\title{
Wide-bandwidth, low-loss, 19-cell hollow core photonic band gap fiber and its potential for low latency data transmission
}

\author{
N. V. Wheeler, M. N. Petrovich, R. Slavík, N. Baddela, E. Numkam, J. R. Hayes, D. R. Gray, \\ F. Poletti and D. J. Richardson \\ Optoelectronics Research Centre, University of Southampton, Highfield Campus, SO17 1BJ, Southampton, UK \\ nvw1v10@orc.soton.ac.uk
}

\begin{abstract}
A record low loss $(3.5 \mathrm{~dB} / \mathrm{km})$ for a wide operating bandwidth HC-PBGF is reported. Detailed time-of-flight measurements are also presented, enabling first measurements of latency and differential group delay between mode groups in HC-PBGF.

OCIS codes: (060.5295) Photonic crystal fibers; (060.2330) Fiber optics communications
\end{abstract}

\section{Introduction}

Hollow core-photonic bandgap fibers (HC-PBGFs) have many intriguing properties including ultralow nonlinearity and low latency relative to conventional (solid) forms of optical fiber. As a consequence they are of great interest as a potential transmission medium in next-generation optical communication systems. However, in order to have any chance of competing with conventional transmission fibers, the possibility of achieving low loss over a suitably extended bandwidth needs to be demonstrated. Moreover, since loss in such fibers is ultimately limited by scattering at the silica/air core interface, ultralow loss will only ever be achieved by moving to core designs that are inherently multi-mode (MM). Hence it is evident that the ability to reliably control and manage modal content within such fibers will be a further fundamental requirement if such fibers are ever to be useful in long haul optical fiber communications. To date the current state-of-the-art in terms of loss for a HC-PBGF is $1.7 \mathrm{~dB} / \mathrm{km}$ at $1565 \mathrm{~nm}$ as reported by Mangan et al. in 2004 [1]. This record low loss was achieved in a 19-cell defect core using an antiresonant core surround to minimize overlap of the fundamental mode (FM) with the silica/air core interface. However, use of such a thick core wall surround introduces many surface modes (SMs) [2] within the low loss bandgap region, which not only increases loss at specific wavelengths (thus significantly decreasing the usable bandwidth to $<20 \mathrm{~nm}$ ), but also degrades the modal stability and polarization properties of the fibers. Therefore, despite the headline record loss, this fiber design is not suitable for the fabrication of HC-PBGF for communications. On the other hand, a HC-PBGF with a minimum loss of $9.5 \mathrm{~dB} / \mathrm{km}$ and a wide bandwidth ( 175 $\mathrm{nm} 3 \mathrm{~dB}$ width) using a 7-cell defect core with a thin-walled core surround (50\% thickness of the cladding struts [2]) has been reported [3]. Theoretically, the same thin-walled core design concept can be applied to 19-cell fibers offering reduced FM overlap with the core surround and thus reduced losses [4]. This requires extremely precise control of the core/cladding interface which becomes increasingly difficult as the core size increases. Consequently, all 19-cell core fibers reported to date support several SMs due to inhomogeneities in the core-wall thickness and distortions in the first ring of the cladding structure.

Here we report for the first time a 19-cell HC-PBGF which simultaneously provides for low loss $(3.5 \mathrm{~dB} / \mathrm{km})$ and a wide transmission bandwidth (160 nm $3 \mathrm{~dB}$ width). Furthermore, we present the results of (spectrallyresolved) Time-of-Flight (TOF) experiments using short pulses which allow us to assess the modal content within our fiber under different excitation conditions and to measure both the intermodal and chromatic dispersion of the various fiber modes observed. Our measurements indicate low mode coupling and thus the possibility of transmitting data on just one or a few optical modes of these complex multimode structures. Finally, measuring absolute TOF, we show for the first time as best we are aware, low latency pulse transmission through a HC-PBGF.

\section{Fiber Fabrication}

The 19-cell HC-PBGF was fabricated using the conventional stack and draw technique. No central core tube was used in order to avoid increasing the fiber core wall thickness and to minimize surface mode guidance in the bandgap region. However, due to small residual core surround distortions, SMs could not be completely eliminated and typically two groups of SMs were observed in short lengths of the fiber. Through an extensive study of draw parameters it was found that the spectral location of these SMs could be controlled and repeatably pushed close to the edges of the bandgap. Careful control of the core and cladding expansion ratios was essential and achieved by accurately defining and maintaining the differential pressurization between these two regions during the second stage draw from cane to fiber. The fabricated fiber has $6 \frac{1}{2}$ rings of cladding holes with an average pitch and relative hole size of $4.4 \mu \mathrm{m}$ and 0.97 respectively, and a core size of $26 \mu \mathrm{m}$ (Fig. 1(a), inset). Its measured loss, obtained by 
a careful cutback from $271 \mathrm{~m}$ to $10 \mathrm{~m}$ is shown in Fig. 1(a) together with a plot showing the lowest reported measured loss in a HC-PBGF to date extracted from Mangan et al. [1]. A minimum loss of $3.5 \mathrm{~dB} / \mathrm{km}$ at $1500 \mathrm{~nm}$ and a very wide surface mode free central region with $3 \mathrm{~dB}$ transmission bandwidth of $160 \mathrm{~nm}$ were achieved. Note that this may be an overestimate of the FM loss due to residual content of lossier high order modes (HOMs), especially at the short cut-back length. Figure 1(b) shows finite element method calculations of the loss components for an ideal fiber with the same cladding but no SMs at all. Here the confinement loss is obtained from the imaginary part of the propagation constant, the scattering loss is estimated through the fiber's F-parameter [5], previously calibrated with a reference fiber, while the total loss is the sum of the two. The excellent agreement indicates that in the central region of the bandgap the loss is dominated by surface scattering, and only towards the edges does the confinement loss play a role. As is well known, scaling the central wavelength of the fiber to $\sim 2 \mu \mathrm{m}$ should reduce the loss by a factor of two relative to a fiber designed for $1.55 \mu \mathrm{m}$ operation [5].
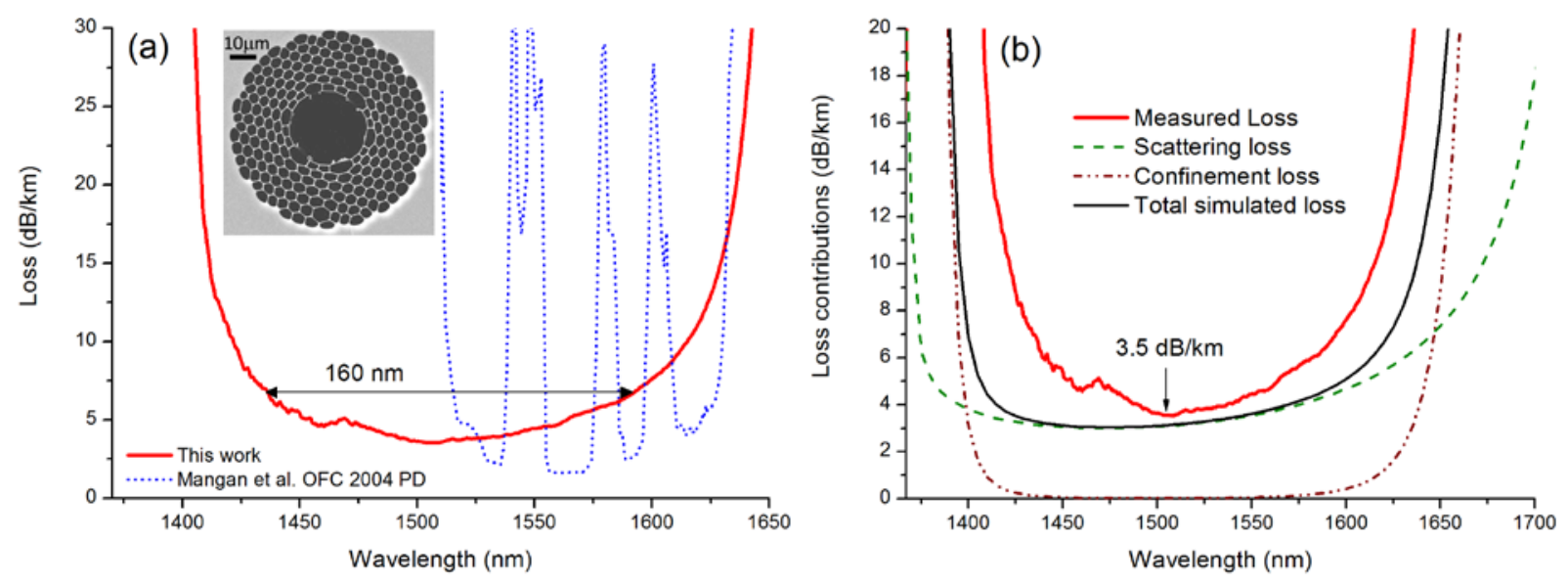

Figure 1: (a) Cut-back loss measurement of the HC-PBGF fabricated in this work (SEM in the inset), as compared to the $1.7 \mathrm{~dB} / \mathrm{km}$ record low loss HC-PBGF of [1] (b) Simulated confinement, surface scattering and total loss contributions of an ideal, surface mode free fiber with the same cladding, compared to the measured loss.

\section{Time-of-flight Measurements}

Large core HC-PBGFs support a multitude of modes: 19-cell fibers in particular are expected from simulations to guide around 40 modes (including degeneracies) [6], although to the best of our knowledge no detailed experimental characterization of their high-order modal content or properties has been performed to date. In order to gain some insight into their complex modal behavior and to study their differential group delay (DGD) we set-up a TOF experiment using a passively mode-locked fiber laser (Calmar) producing sub ps pulses tunable in the C-band and at a repetition rate of $20 \mathrm{MHz}$. The pulse bandwidth was reduced to $2 \mathrm{~nm}$ FWHM using a bandpass filter with a Gaussian-shaped passband, which for chirp-free Gaussian pulses corresponds to a pulse duration of 2-3 ps. The pulses are launched into $260 \mathrm{~m}$ of the HC-PBGF via butt-coupling with an endlessly single mode fiber (LMA-15 from NKT Photonics), which has a mode field diameter (MFD) of $12 \mu \mathrm{m}$, reasonably matched to that of the HCPBGF $(16 \mu \mathrm{m})$. At the output the HC-PBGF is directly inserted into the multimode optical port of a $10 \mathrm{GHz}$ bandwidth sampling oscilloscope, electrically triggered by the laser source.

When deliberately offsetting the launch, several HOMs are efficiently excited at the HC-PBGF input and due to their lower group velocity relative to the FM they produce delayed peaks in the oscilloscope traces. Real time information of the relative peak position and amplitude as a function of launch conditions can be observed in this way. Figure 2(a) shows, for example, that with a launch offset by $12 \mu \mathrm{m}$ from the central position 9 distinct peaks can be observed for 2 different input polarizations. By cross-comparing their DGD with numerical simulations we can identify them as $\mathrm{LP}_{11}$ (delayed by $\sim 7-10 \mathrm{ps} / \mathrm{m}$ from the $\left.\mathrm{LP}_{01}\right), \mathrm{LP}_{21}(\sim 18-19 \mathrm{ps} / \mathrm{m}), \mathrm{LP}_{02}(\sim 21-23 \mathrm{ps} / \mathrm{m})$ and $\mathrm{LP}_{31}$ ( $31-34 \mathrm{ps} / \mathrm{m})$. The red and black traces, corresponding to different input polarizations, show that good mode control can be achieved by selectively exciting different vector modes within a mode group and their high extinction ratio seems to indicate relatively low intermodal cross-talk. Furthermore, measurements in the 1530-1560 nm range (Fig.2(c)) indicate no SM interaction even for HOMs and yield the dispersion of all modes. By choosing suitable launch conditions a similar amount of power can be achieved in $\mathrm{LP}_{11}$ and $\mathrm{LP}_{01}$ modes at the fiber output. Figure 2(b) shows the same measurement with an optimized central launch. In this case, all HOMs other than the azimuthally symmetric $\mathrm{LP}_{02}$ were suppressed by more than $22 \mathrm{~dB}$, the maximum dynamic range of our set-up (which was limited by the noise properties and impulse response of the receiver used, see green trace in Fig. 2(b)). The $\sim 17 \mathrm{~dB}$ 
extinction ratio of the $\mathrm{LP}_{02}$ mode increased to $>30 \mathrm{~dB}$ once a butt-coupled SMF pigtail was introduced to spatially filter the HC-PBGF output. No evidence of distributed cross-talk could be observed within the dynamic range of our set-up which is extremely promising in terms of potential use for single mode data transmission.

Finally we measured the absolute TOF through the HC-PBGF. As a source for this we used an acousto-optic modulator driven by an arbitrary waveform generator to carve $\sim 150$ ns pulses at a repetition rate of $100 \mathrm{kHz}$ from a CW source operating at the center of the bandgap $(1556 \mathrm{~nm})$. The real time oscilloscope traces for pulses through $260 \mathrm{~m}$ of our HC-PBGF (red), the same length of a reference telecoms NZ-DSF fiber (blue) and in the back-to-back configuration (black) are shown in Fig. 2(d). The measurements indicate group velocities (group indices) of $290 \mathrm{~m} / \mathrm{ps}$ (1.003) for the HC-PBGF, as compared to $205 \mathrm{~m} / \mathrm{ps}$ (1.464) for the standard telecoms fiber. This confirms that due to a $>99.8 \%$ overlap of the guided modes with air, light in the HC-PBGF propagates at group velocities close to the speed of light in vacuum, about 1.46 times faster than in a solid silica fiber. Therefore HC-PBGFs should enable data transmission with approximately $1.54 \mu \mathrm{s} / \mathrm{km}$ less latency than conventional fibers.
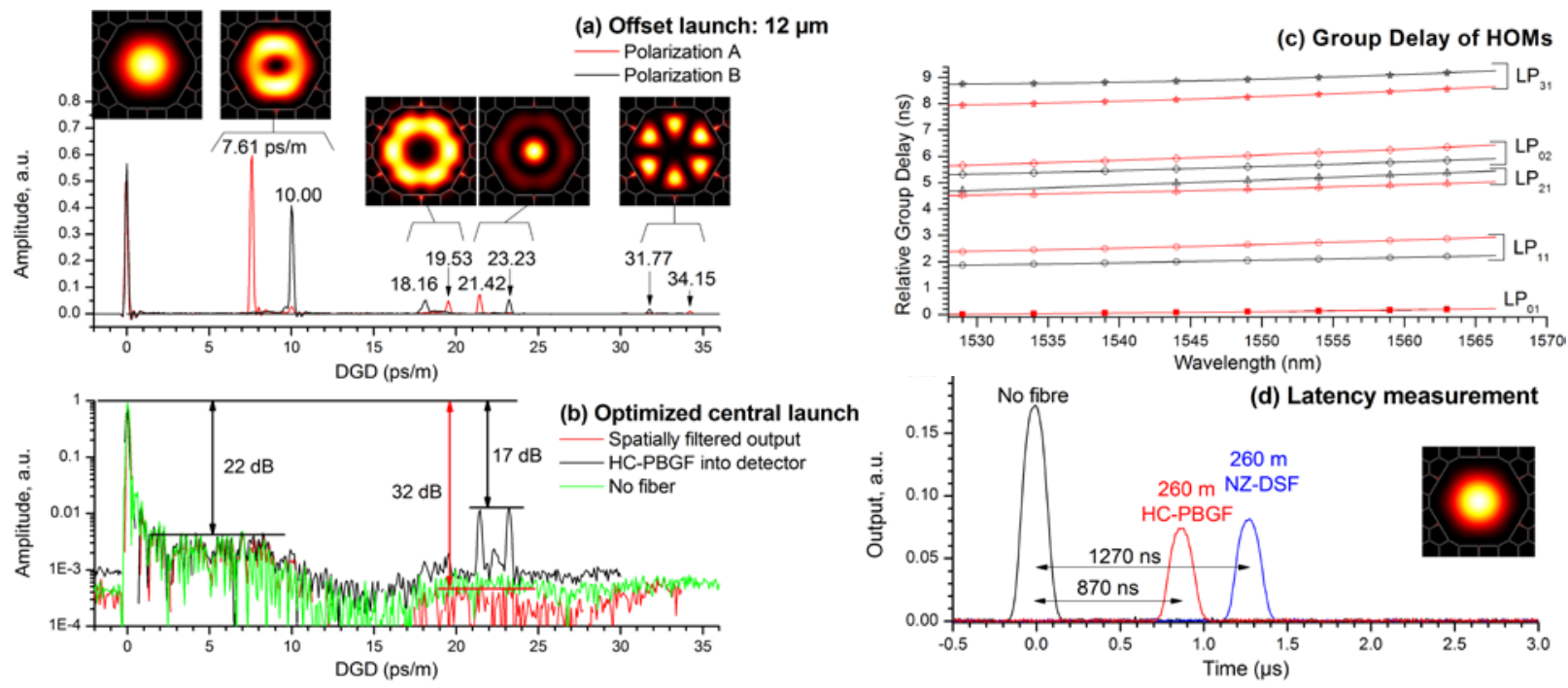

Figure 2. TOF measurements: (a) launch with a $12 \mu \mathrm{m}$ lateral offset: 5 distinct mode groups can be observed and their relative intensities adjusted by changing launch conditions. The simulated modal intensities are also shown; (b) optimized central launch achieving > 22 dB extinction of any HOM; (c) Wavelength dependence of HOM group delay; (d) latency measurement.

\section{Conclusions}

Through reproducible control of surface modes in a large core, multimode 19-cell HC-PBGF we report a fiber with a record combination of low loss $(3.5 \mathrm{~dB} / \mathrm{km})$ and wide $3 \mathrm{~dB}$ bandwidth $(160 \mathrm{~nm})$ at $1500 \mathrm{~nm}$. Time-resolved measurements show the presence of several well-behaved HOMs with no readily observable intermodal coupling, highlighting the potential for single-mode/few-mode data transmission. First group velocity measurements confirm $\sim 1.46$ times faster signal propagation than in conventional fibers, a feature of great interest for low latency telecoms. Although the current experiments were performed in relatively modest lengths of fiber and a host of further scientific and more practical challenges clearly remain, we consider our results to represent an important step forward in understanding the modal properties of PBGFs and towards establishing the viability of using these fibers for data transmission. Based on these results, we believe that by further structural and process optimisation there is a realistic prospect of further reducing losses in these wide bandwidth HC-PBGFs down to the 0.1-0.4 dB/km level at $2 \mu \mathrm{m}$ wavelengths. This work was supported by the EU 7th Framework Programme under grant agreement 228033 (MODE-GAP) and by the UK EPSRC through grants EP/I01196X/1 (HYPERHIGHWAY) and EP/H02607X/1.

\section{References}

[1] B. J. Mangan, et al., "Low loss (1.7 dB/km) hollow core photonic bandgap fiber," OFC 2004, PD24.

[2] R. Amezcua-Correa, et al., "Optimizing the usable bandwidth and loss through core design in realistic hollow-core photonic bandgap fibers," Optics Express, vol. 14, pp. 7974-7985, 2006-07 2006.

[3] R. Amezcua-Correa, et al., "Control of surface modes in low loss hollow-core photonic bandgap fibers," Optics Express, vol. 16, pp. 11421149, Jan 212008.

[4] R. Amezcua-Correa, et al., "Design of 7 and 19 cells core air-guiding photonic crystal fibers for low-loss, wide bandwidth and dispersion controlled operation," Optics Express, vol. 15, pp. 17577-17586, Dec 242007.

[5] P. J. Roberts, et al., "Ultimate low loss of hollow-core photonic crystal fibres," Optics Express, vol. 13, pp. 236-244, Jan 102005.

[6] M. N. Petrovich, et al., "Robustly single mode hollow core photonic bandgap fiber," Optics Express, vol. 16, pp. 4337-4346, $2008-032008$. 